\title{
Advanced Devices \& Instrumentation: Integrated for Functionality to Change the World
}

\author{
Wei Wang ${ }^{1}$ and Daping $\mathrm{Chu}^{2}$ \\ ${ }^{1}$ China Aerospace Science and Technology Corporation, China \\ ${ }^{2}$ University of Cambridge, UK \\ Correspondence should be addressed to Daping Chu; dpc31@cam.ac.uk \\ Received 11 February 2020; Accepted 11 February 2020; Published 4 April 2020 \\ Copyright (c) 2020 Wei Wang and Daping Chu. Exclusive Licensee Beijing Institute of Aerospace Control Devices. Distributed \\ under a Creative Commons Attribution License (CC BY 4.0).
}

Science and engineering research in the world has been moving more and more towards the interactions and interchanges between different subject fields and different system levels in recent years, in searching for innovation and new functionalities. This is the area where scientific breakthroughs and disruptive technologies emerge and change the world. An endeavour of this kind often needs a huge amount of resource and manpower over a considerable period of time to accomplish. However, it is not easy to find a place to report new developments because conventional journals divide along established disciplines, not the interlinks among them, and they focus on finished works not progressive outcomes. A journal which is dedicated to such an emerging trend is clearly required.

The integration of advanced devices and instrumentation for functionalities is a cornerstone for the development of science and technology. It is a main approach of scientific research for the exploration of the unknown world and universe. At the same time, it exists in our everyday life, as a platform technology to create new functions and new capabilities to improve people's ways of living. All these involve interlinked research activities at every level, from novel devices and sensors, functional instrumentation, intelligent information integration and processing, ubiquitous internet and internet of things, and performance testing and metrology. They are essential to basic research as well as technology development for all kinds of applications addressing technical challenges to social problems and societal needs, such as modern manufacturing, healthcare, renewable energy, emis- sion control, environmental monitoring, and smart cities and infrastructures. Undoubtedly, Advanced Devices \& Instrumentation is at the heart of modern science and engineering and their future development, which has been having an enormous social and economic impact on society over the years.

This newly established Science Partner Journal (SPJ) "Advanced Devices \& Instrumentation" (ADI) is a joint effort in response to such a need, through the close collaboration between Beijing Institute of Aerospace Control Devices (BIACD) and American Association for the Advancement of Science (AAAS). It will provide a high-quality open access platform with a special focus on integration and functionalities to serve scientists and engineers worldwide, as a showcase of important academic breakthroughs and key application progresses from devices to instrumentation at each stage of the research from the beginning to end.

Research activities in the targeted areas of ADI (see the Mission and Scope of the journal) are all over the world, and the same is true for the authorship and readership. The main attraction to the potential authors is ADI's uniqueness in interlinking the advanced devices and novel functionality of integrated systems as well as, in the long run, the high impact in the field. The ADI Editorial Office is aimed at providing an unparalleled author service from day one with friendly guidance and support all the way through the publication process, such as providing a time-limited fast review process, immediate online publication after acceptance, and editorial help for publication. The day-to-day operation of 
the journal will be managed by a dedicated editorial team with its main office at BIACD in Beijing and additional support from Centre for Advanced Photonics and Electronics (CAPE) in Cambridge, to ensure effective and quick response to potential authors from around the world. Additional services such as promotion of published papers on multimedia platforms, prompts of publications of related papers, citations of the published papers, and professional conferences and events will be provided over time. The overall objective is to build a friendly ecosystem with ADI as its focal point, for the authors, readers, scientists, engineers, and those who are interested in this area to come back and exchange their ideas, results, views, and comments once they encounter an ADI publication. For further information, please contact the ADI Editorial Office by email: adiscience@sina.com at any time.

\section{Conflicts of Interest}

The authors declare that they have no conflicts of interest.

Wei Wang

Daping Chu 